\title{
Incidence and features of heparin- induced thrombocytopenia in mechanical heart valves and immunologic cardiac diseases
}

\author{
Manzoor H Mangi ${ }^{1 *}$, Ulrich Stock², Kanval Ali', Eftychia \\ Galiatsou ${ }^{3}$, Visuvanathan Jeyakumar ${ }^{1}$, Ana Hurtado Doce ${ }^{3}$, \\ Paul Harris ${ }^{4}$ and Sunil K Bhudia ${ }^{2}$
}

1Department of Hematology, Harefield Hospital, Hill End Rd, Harefield, London Uxbridge UB9 6JH, UK ${ }^{2}$ Department of Cardiothoracic Surgery, Harefield Hospital, Hill End Rd, Harefield, London Uxbridge UB9 6JH, UK

${ }^{3}$ Department of Intensive Care Medicine, Harefield Hospital, Hill End Rd, Harefield, London Uxbridge UB9 6JH, UK

${ }^{4}$ Department of Anesthesia, Harefield Hospital, Hill End Rd, Harefield, London Uxbridge UB9 6JH, UK

\section{Abstract}

Background: Heparin-induced thrombocytopenia/thrombosis (HIT/T) is characterized by a fall in platelet count 5-10days after starting heparin therapy and is diagnosed with specific 4-T clinical features and laboratory tests. This complication is relatively common in Cardiothoracic surgery patients.

Objective: To evaluate the positive and negative predictive value of various HIT laboratory tests and assess any correlation between HIT, the underlying diagnosis, underlying procedure, and mechanical cardiac devices.

Patients and methods: The patient's medical records were correlated with two laboratories HIT diagnostic tests, the pan-specific screening test with IgG, IgA, and IgM antibodies, followed by HIT specific IgG ELISA.

Results: Total $n=80$ patients were assessed, $48 \%(n=38)$ were HIT screen pan-specific negative and $50 \%(n=40)$ were HIT pan-specific positive and 2 cases were inconclusive. $17 \%$ $(n=14)$ were both pan-specific and specific HIT IgG ELISA positive. There were 5 atypical cases. One patient had Eosinophilic myocarditis and was HIT ELISA IgG neg. Argatroban was given on clinical grounds with successful recovery. One patient with Sarcoidosis had an aggressive course and received IV Immunoglobulin (IVIG) but succumbed secondary to liver failure. One patient progressed to gut ischemia and had surgical intervention but succumbed. Two patients with mechanical heart valves were on Argatroban but relapsed and responded to IVIG therapy.

Conclusion: Our study indicates that $9 / 16$ ( $>50 \%$ ) HIT-positive patients had valve replacement or cardiac devices suggesting that like knee arthroplasty there is a high incidence of HIT in patients with mechanical heart valves and cardiac devices and this warrants further prospective study.

\section{Introduction}

Heparin-induced thrombocytopenia (HIT) is characterized by a rapid fall in the platelet count, normally 5-10 days after starting heparin therapy with or without thromboembolic complications [1-3]. It is reported that antibodies to the heparin/PF4 complex after cardiac surgery are detected in $25 \%$ to $50 \%$ of patients, but only $35 \%$ show pathological HIT requiring non-heparin anticoagulant [4-6]. This suggests multiple triggering factors of thrombocytopenia and
More Information

*Address for Correspondence:

Dr. Manzoor H Mangi, Department of

Hematology, Harefield Hospital, Hill End Rd, Harefield, London Uxbridge UB9 6JH, UK, Email: mhmangi@aol.com; manzoor.mangi@nhs.net

Submitted: December 21, 2021

Approved: January 04, 2022

Published: January 05, 2022

How to cite this article: Mangi MH, Stock U, Ali K, Galiatsou E, Jeyakumar V, et al. Incidence and features of heparin-induced thrombocytopenia in mechanical heart valves and immunologic cardiac diseases. Ann Clin Hypertens. 2022; 6: 001-008.

DOI: 10.29328/journal.ach.1001028

ORCID: orcid.org/0000-0003-1762-2570

Copyright License: () 2022 Mangi MH, et al. This is an open access article distributed under the Creative Commons Attribution License, which permits unrestricted use, distribution, and reproduction in any medium, provided the original work is properly cited.

Keywords: Heparin Induced Thrombocytopenia (HIT); Thrombosis; Cardiothoracic surgery; Mechanical heart valves (MVR); Immune Thrombocytopenia (ITP)

\section{(D) Check for updates \\ OPEN ACCESS}


laboratories and these were not available in this retrospective study. To this end, we have done 1-year, retrospective study to assess the laboratory diagnosis for detecting anti-PF4/ heparin antibodies and assays in cardiothoracic intensive care unit (ICU) patients. To evaluate the positive and negative predictive value of various HIT laboratory tests, and any pathogenic triggering correlation between HIT, spontaneous HIT, the underlying diagnosis, and underlying cardiothoracic procedure.

\section{Patients and methods}

This is a retrospective 1-year, single-center cardiothoracic Intensive care Unit (ICU) study. Clinical details were obtained from EPR (electronic patient records, Graphnet Carecentric software (Graphnetnet Health Ltd), ICU-IntelliSpace Critical Care and Anesthesia (ICCA) software system (Philips). The ' 4 T's' (Thrombocytopenia, Timing of platelet count fall, Thrombosis and exclude other causes for Thrombocytopenia,) was used for the clinical diagnosis of HIT. Laboratory data were collected from the pathology Win-Path system (Winpath software, CliniSys). Two laboratory diagnostic tests were performed, the pan-specific screening test which includes pan-specific antibodies IgG, IgA, and IgM antibodies. This was performed by HemoSIL HIT-Ab (PF4-H) antibodies by latex particle-enhanced immunoturbidimetry performed by ACL500. Confirmatory HIT mono-specific, IgG HIT was done by Chemiluminescent immunoassay (HemoSIL AcuStar HIT-
IgG) performed on AcuStar. All equipment and HIT reagents were from Instrumentation Laboratory (A Werfen Company) and tests were done as per manufacturers and company specifications. None of the patients had a history of COVID-19 infection or COVID-19 vaccination [9]. Platelet Functional assays (PFA), the serotonin release assays (SRA), and heparininduced platelet aggregation assays (HIPA) were not available in this retrospective study.

\section{Results}

80 patient records with HIT probability " 4 T" score $>4$ was available for assessment. Male $n=57$ and female $n=23$, median age around 56 years (range 30 - 75). Pan-screen HIT IgGAM screen was positive in $n=38(48 \%)$ and negative in $n=40(50 \%)$ patients. Only $n=14$ patients $(17 \%)$ were both pan-specific and HIT IgG ELISA positive and 2 cases were inconclusive.

All HIT positive acute patients received Argatroban except two cases who received Fondaparinux. Clinical and laboratory details are summarized in Table 1.

9/16 (> 50\%) HIT-positive patients had valve replacement or cardiac devices.

There were $4 / 16(25 \%)$ deaths, two were unrelated to HIT, one may be transplant failure, but $1 / 16(6.2 \%)$ were attributed to HITT with gut ischemia. 5/16 (31\%) cases had a

Table 1: Clinical Details of Cardiothoracic HIT-Positive patients.

\begin{tabular}{|c|c|c|c|c|c|c|c|c|}
\hline Pt no: & Age/sex & Diagnosis & HIT- IgG- ELISA & Bleeding & Thrombosis & ECMO & $\begin{array}{l}\mathrm{A} / \mathrm{C}+\text { Other } \\
\text { therapy }\end{array}$ & Outcome \\
\hline 1 & $63 / \mathrm{M}$ & t-MVR+t-AVR & pos & No & No & No & $A+W$ & FU \\
\hline 2 & $54 / \mathrm{M}$ & $\begin{array}{l}\text { Hypertrophic obstructive } \\
\text { Cardiomyopathy, AF, TIA }\end{array}$ & pos & yes & Yes-LV thrombus & yes & A & RIP \\
\hline 3 & $63 / \mathrm{M}$ & $m-M V R+m-A V R$ & pos & No & Yes-LA thrombus & Yes & $A+W+I V I G$ & FU \\
\hline 4 & $57 / M$ & VAD & pos & No & No & - & A & FU \\
\hline 6 & $30 / \mathrm{M}$ & VAD & pos & & No & - & A & FU \\
\hline 7 & $53 / \mathrm{M}$ & m-MVR & pos & yes & No & Yes & $A+W$ & FU \\
\hline 8 & $67 / M$ & Bilateral lung transplant & pos & Yes & No & Yes & A & RIP \\
\hline 9 & $45 / \mathrm{M}$ & $m-A V R$ & pos & No & Yes RAthrombus & Yes & $A+W$ & FU \\
\hline 9 & $66 / \mathrm{M}$ & $\begin{array}{l}\text { Late presentation STEMI, PCI, not suitable for } \\
\text { heart transplant/for alternative treatment }\end{array}$ & pos & Yes & No & Yes & A & RIP \\
\hline 10 & $75 / F$ & $\begin{array}{c}\text { Type A aortic dissection, ascending aorta } \\
\text { (28mmHaemashield } \\
\text { graft) and resuspension of the } \\
\text { Aortic Valve }\end{array}$ & pos & No & No & No & $F+D$ & FU \\
\hline 11 & $74 / \mathrm{M}$ & Aortic+mitral+tricuspid repair and CABG & pos & No & No & No & $A+D$ & FU \\
\hline 12 & $56 / \mathrm{M}$ & $\mathrm{t}-\mathrm{MVR}+\mathrm{CABG}$ & pos & No & No & No & $\mathrm{F}$ & FU \\
\hline 13 & $55 / \mathrm{M}$ & Sarcoidosis-cardiac transplant & Neg & Yes & No & $\begin{array}{l}\text { ECMO } \\
\text { lines }\end{array}$ & $A+I V I G$ & RIP \\
\hline 14 & $56 / F$ & $\begin{array}{l}\text { Eosinophilic } \\
\text { Myocarditis }\end{array}$ & Neg & No & $\begin{array}{l}\text { Yes. } P E+L \\
\text { legischemia }\end{array}$ & Yes & $\begin{array}{c}\text { Myc+MP and } \\
\text { A+D }\end{array}$ & FU \\
\hline 15 & $55 / \mathrm{M}$ & $\begin{array}{l}\text { t-AVR and CABG. Past H/O ITP in remission for } \\
\text { 2-years. }\end{array}$ & pos & No & $\begin{array}{c}\text { Yes. } \\
\text { PE+gutischemia }\end{array}$ & yes & A & RIP \\
\hline 16 & $56 / F$ & Aortic intramural haematoma repair and m-AVR & pos & No & No & No & $A+W$ & FU \\
\hline
\end{tabular}

Short terms used: HIT: Heparin Induced Thrombocytopenia/thrombosis (HITT); ITP: Immune Thrombocytopenia; A/C: Anticoagulants. A: Argatroban; W: Warfarin; F: Fondaparinux; D: Direct Oral Anticoagulants (DOACS); Myco: Mycophenolate; MP: Methylprednisolone; IVIG: Intravenous Immunoglobulin S; Pos: Positive; Neg: Negative; FU: Follow Up; RIP: Deceased; VAD: Ventricular Assisted Device; MVR: Mitral Valve Replacement; AVR: Aortic Valve Replacement; t: tissue and m: mechanical; CABG: Coronary Artery Bypass Graft; STEMI: ST Elevation Myocardial Infarction; ECMO: Extracorporeal Membrane Oxygenation; PCI: Percutaneous Coronary Intervention; ICCA: IntelliSpace Critical Care and Anesthesia; Critical Care Information System; ELISA: Enzyme-Linked Immunoassay; PFA: Platelet Function Assay; HIPA: Heparin Induced Platelet Aggregation; SRA: Serotonin Release Assay 
typical clinical presentation, laboratory features, and clinical course. Figure 1 shows the typical clinical course of a patient with post mechanical mitral valve surgery complicated by HIT and LA thrombus, responded to the non-heparin anticoagulant, Argatroban. This was later changed to Warfarin before discharge.

Two patients with post mechanical heart valve surgery had the unexpected course. Patients were on Argatroban but relapsed during ICU stepdown stage. One developed severe thrombocytopenia and LA thrombus and responded to IV immunoglobulin (Figure 2). The other one had mild thrombocytopenia and had spontaneous recovery and changed to Warfarin.

One patient had Eosinophilic myocarditis diagnosed on cardiac biopsy and was HIT ELISA IgG neg but had a high 4-T score, pulmonary thrombosis, and left leg ischemia requiring three embolectomies. Immunology investigations showed C3: $179 \mathrm{mg} / \mathrm{dL}, \mathrm{C} 4: 26.3 \mathrm{mg} / \mathrm{dL}$, IgA $2.4 \mathrm{~g} / \mathrm{L}$, IgG $11.9 \mathrm{~g} / \mathrm{L}, \mathrm{IgM} 0.6 \mathrm{~g} / \mathrm{L}$. All within normal range. Rheumatoid factor and anticardiolipin antibodies were negative. IgE was raised to $78.1 \mathrm{KU} / \mathrm{L}$ (NR 0-75). Hypereosinophilic syndrome (HES) Fluorescence in situ hybridization (FISH) myeloid gene panel was also performed on peripheral blood. Interphase FISH analysis of 100 cells showed the presence of a normal signal pattern for the FIP1L1/CHIC2/PDGFRA (4q12), PDGFRB (5q32), FGFR1 (8p11), JAK2 (9p24), ABL1 (9q34.1), and BCR (22q11.2) loci with no available genetic evidence of Hypereosinophilic syndrome (HES). The patient had primary eosinophilic myocarditis and was on extracorporeal membrane oxygenation (ECMO) and received high dose methylprednisolone and Mycophenolate Mofetil (MMF) for Eosinophilic myocarditis and non-heparin anticoagulant
Argatroban for ECMO and multiple thrombi. The patient responded with platelet recovery after stopping Heparin and on receiving Argatroban. This was full recovery with high dose methylprednisolone and MMF and argatroban with no further thromboembolic episodes. Argatroban was changed to direct oral anticoagulants (DOACS) on discharge. This patients' diagnostic and clinical course and therapy are illustrated in Figure 3.

One patient with Sarcoidosis had the resistant course and received IV IG but succumbed secondary to transplant-related and liver issues.

One patient with tissue aortic valve replacement (AVR) and coronary artery bypass graft (CABG), had a history of immune thrombocytopenia treated with steroids and was in remission for 2-years, pre-cardiothoracic surgery. This patient had the remitting and relapsing course. The patient developed HIT with pulmonary thrombosis post cardiothoracic surgery and on ECMO initially responded to stopping Heparin and starting Argatroban therapy. This case then relapsed while on Argatroban and progressed to severe thrombocytopenia and gut ischemia with refractory HIT which was negative for panspecific and HIT IgG antibodies.

The patient had surgical intervention but became palliative and passed away before receiving immunotherapy. This clinical course is illustrated in Figure 4.

Interconnected pyramids, a modified iceberg model, and interconnected block processes illustration (Figure $5 \mathrm{~A}$ and $\mathrm{B}$ ) summarize laboratory features, clinical course, and treatment options for HIT/HITT in post-surgical cardiothoracic cases. Pyramid black base indicating nonHIT causes of

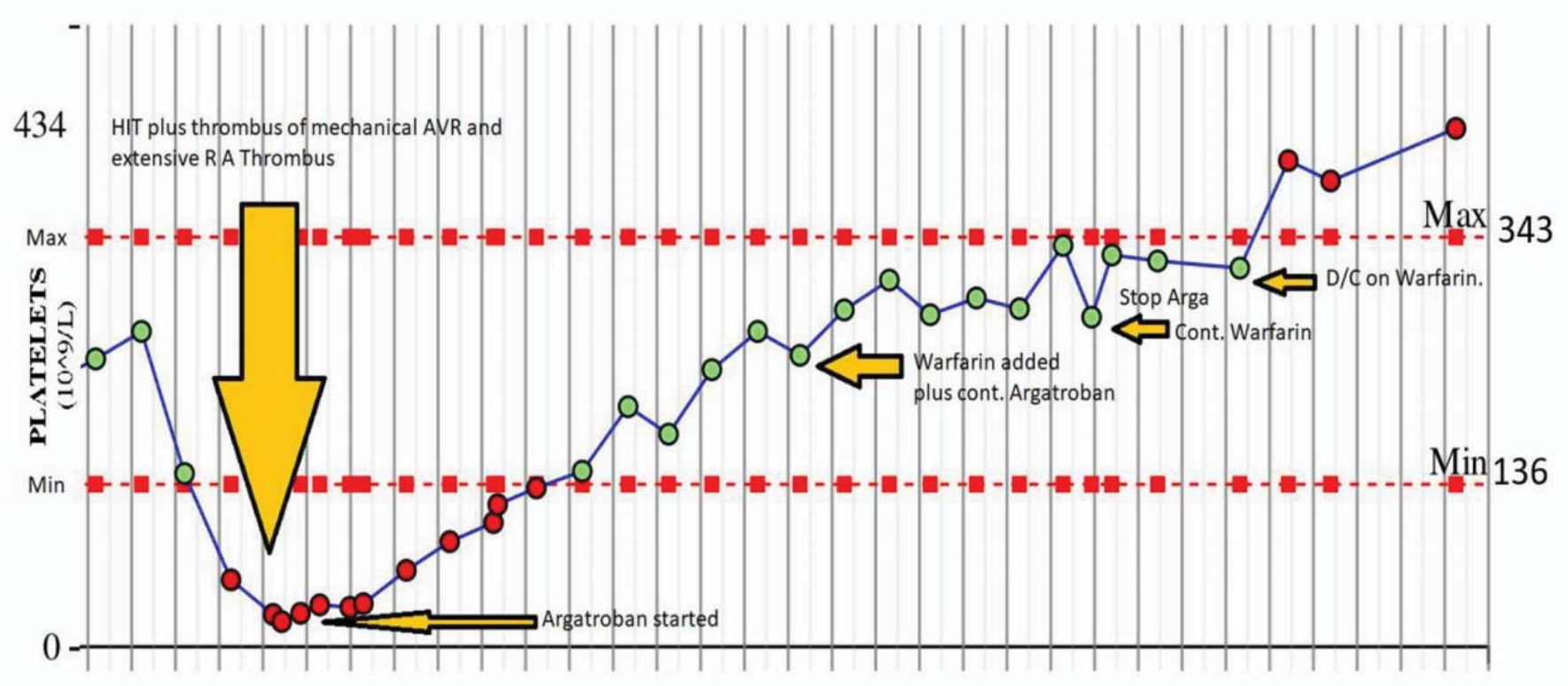

Figure 1: Typical course of HIT: 53-year-old man with mechanical Aortic valve replacement (AVR). The 53-year-old subject had HIT and RA thrombus post-surgery and responded to Argatroban and continuing long-term Warfarin post-surgery. 


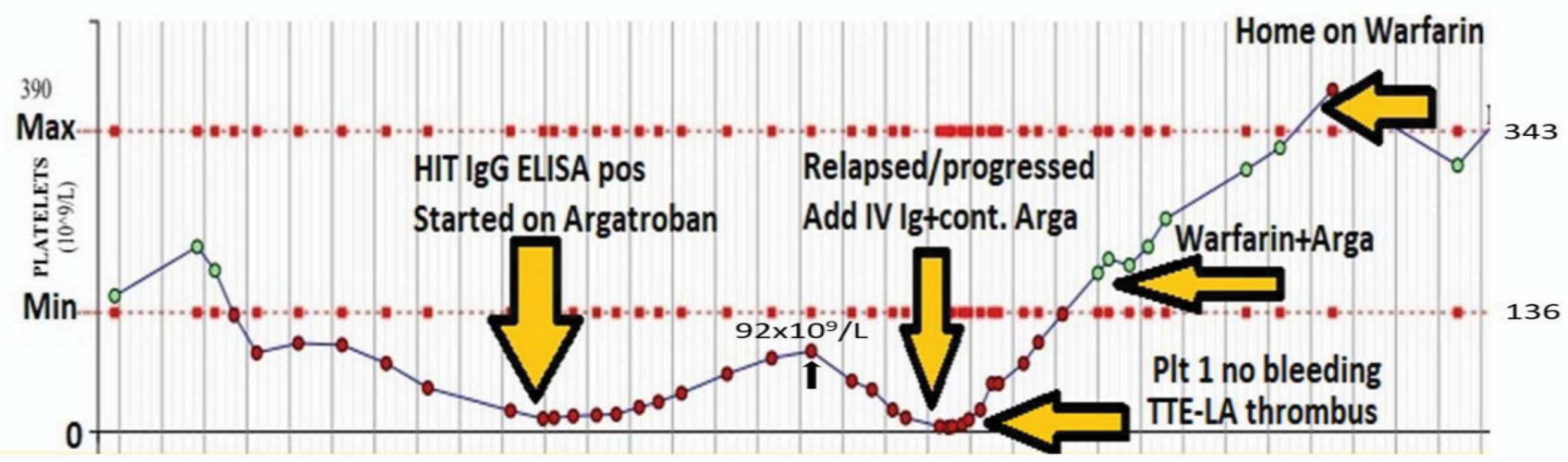

Figure 2: Remitting and relapsing HIT: 63-y male with mitral valve and aortic valve replacement surgery. Development of HIT-complication after urgent mechanical AVR and mechanical MVR on a background of AF and CCF. Patients' HIT relapsed on Argatroban with LA thrombus on Trans Thoracic ECHO-(TTE). The patient was rescued with intravenous immunoglobulin (IV IG) $1 \mathrm{~g} / \mathrm{kg}$ for two days. After discharge patient is continuing on long-term Warfarin.

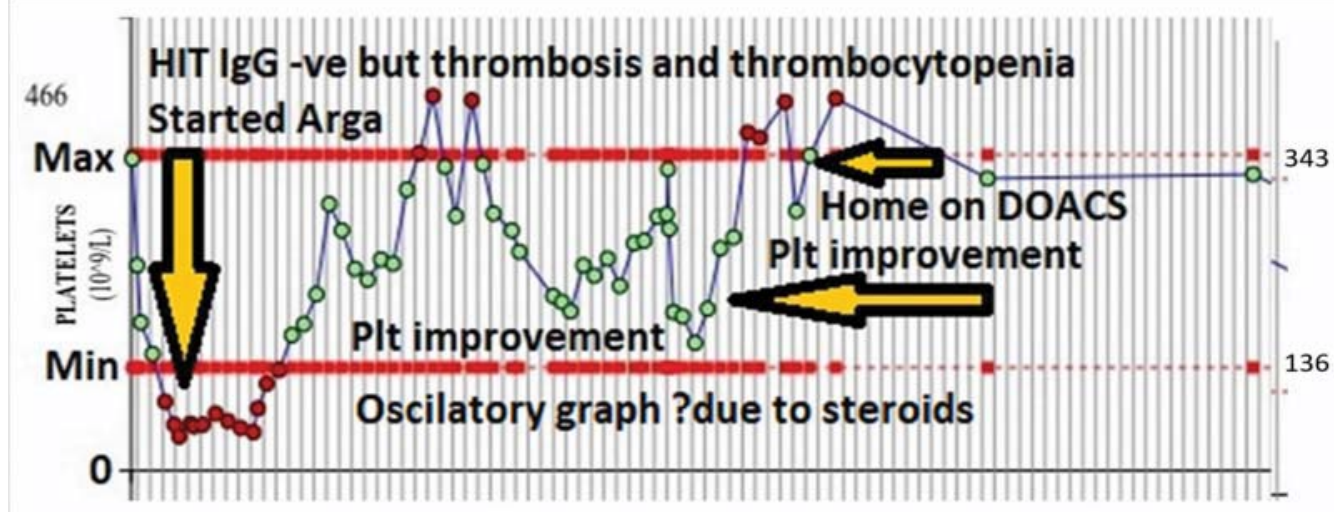

Figure 3: PF4-IgG Antibody negative HIT in Eosinophilic myocarditis: 56-female with Eosinophilic myocarditis on ECMO, developed thrombocytopenia and pulmonary thrombosis and L leg ischemia. HIT-screening-HIT PF-4 lgG negative. No serotonin release assay is available. Commenced on Argatroban on clinical grounds. The patient also received steroids and MMF for eosinophilic myocarditis. Oscillations in the platelet graph may be due to steroid and MMF therapy. Full recovery in platelet count and no further thromboembolism and patient discharged home on DOACS.

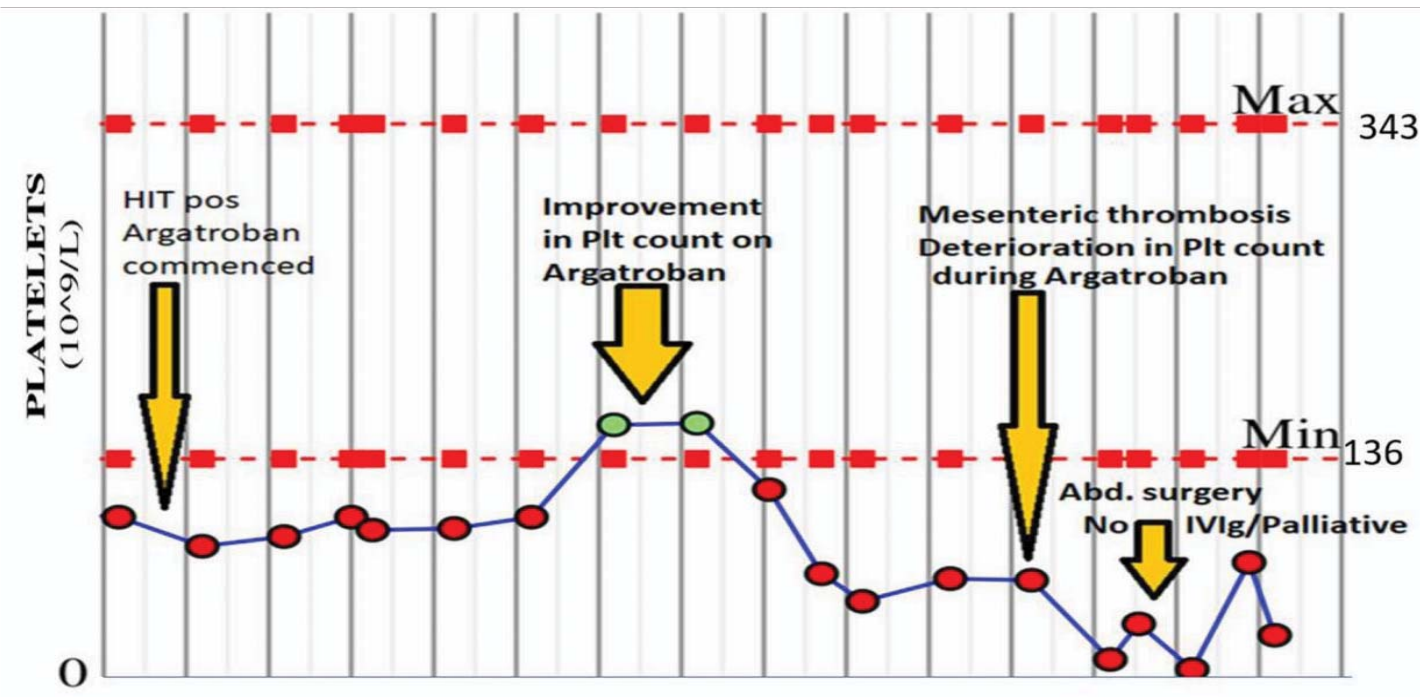

Figure 4: ITP in remission and remitting and relapsing refractory HIT: 55-y male with a past medical history of Immune Thrombocytopenia (ITP) in remission for 2-years. Post cardiac surgery of Tissue AVR plus 2xCABG surgery, on ECMO, with HIT and pulmonary emboli. A complicated course of HIT with the initial response to Argatroban but relapsed with severe and refractory HIT on Argatroban with Bowl ischemia requiring abdominal surgery. Possible cross-over of autoimmune ITP to autoimmune HIT. Repeat HIT antibody screening negative. No Platelet function assays are available. Too unwell, palliative passed away before receiving IV IG. 


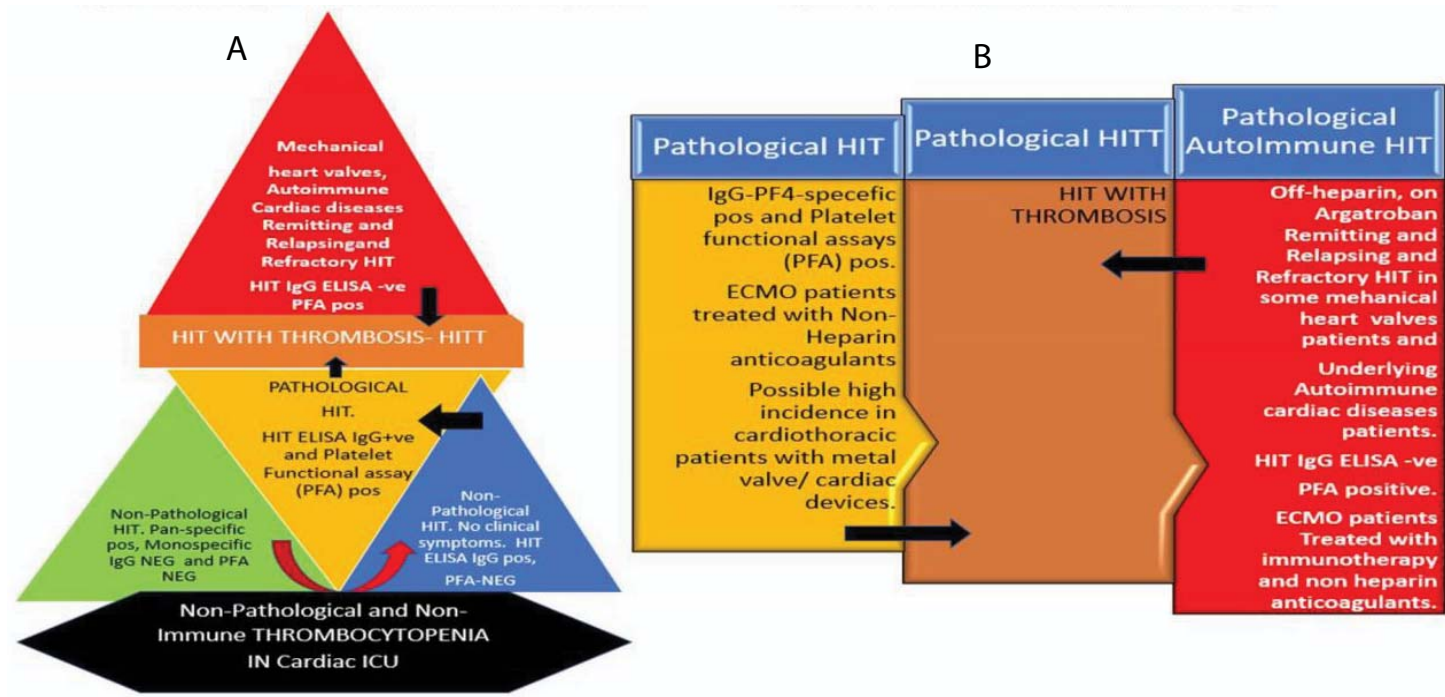

Figure 5: A: Modified diagnostic Iceberg model: Illustration shows different laboratory diagnostic results for cardiothoracic patients. Black zone indicating non-immune and non-HIT causes of thrombocytopenia. Green and blue zones show non-pathological HIT with pan reactive antibodies and some with IgG HIT+ but negative for PFA and no clinical symptoms. Yellow zone shows pathological symptomatic HIT with PFA and HIT IgG positive. The Brown zone indicates HIT with thrombosis and the red zone shows spontaneous immune HIT/T in some mechanical heart valves or cardiac devices or remitting and relapsing HIT on Argatroban. B: Cardiothoracic HIT and treatment options: and immunotherapy interventions. Various arrows show cross-talk and cross-over between various zones in pathologic clinical entities with remitting, progression, spontaneous and relapsing courses.

thrombocytopenia. The green zone displays non-pathological HIT with pan reactive antibodies. Blue zone shows nonpathologic IgG HIT-antibody positivity but negativity for platelet function assays (PFA) and no pathologic HIT. Yellow zone illustrates pathologic symptomatic HIT with PFA and HIT IgG positivity. Brown zone indicates HIT with thrombosis which could be due to pathologic heparin-related HIT or autoimmune or spontaneous HIT. The red zone specifies spontaneous immune HIT or mechanical heart valve triggered or augmented HIT cases with remitting and relapsing HIT course requiring immune intervention.

\section{Discussion}

Our results highlight the complex clinical course of HIT cases in cardiothoracic patients. Various HIT diagnostic tests are important and show variability in positive and negative predictive values. Also, cardiothoracic ICU patients may be thrombocytopenic due to non-immune causes including surgery-related platelet consumption, sepsis and ECMO [4-6]. Our findings suggest that $80 \%$ of thrombocytopenia in ICU was non-immune, secondary, and not HIT-related. These non-HIT causes increase HIT-related laboratory workload. Refining clinical diagnosis and laboratory diagnostic procedures may help in laboratory demand management $[7,11]$. HIT pan-specific antibody test remains the cornerstone of rapid laboratory assessment but it has a positive predictive value of only $35 \%$ in cardiothoracic patients and non-Heparin anticoagulation treatment based on only pan-specific antibody positivity may be uncertain. The usefulness of the PF-4 IgGspecific antibody test in cardiac patients with an autoimmune disease like sarcoidosis and eosinophilic myocarditis remains doubtful. The functional assays SRA or HIPA tests are more specific, and do not detect nonpathogenic, nonactivation HIT antibodies and would be more useful in this cases $[8,11]$. Also, there are some reports of functional assay negative HIT [10]. Therefore, in addition to standard PF4 assays, various PF4-enhanced platelet activation assays, platelet factor 4-dependent P-selectin expression assay (PEA) and hybrid functional assays have been developed in specialized laboratories for robust diagnosis of rare spontaneous unusual, atypical and autoimmune HIT cases [12-14]. The case with eosinophilic myocarditis was interesting. It has typical clinical features of HIT with pulmonary thrombus, leg ischemia and thrombocytopenia after heparin exposure during ECMO. Surprisingly, HIT PF-4 specific IgG test was negative and SRA, HIPA tests, or PF4 enhanced assays were not available during an inpatient stay. Unfortunately, no archival plasma material was available for retrospective analysis. In our patients with eosinophilic myocarditis, pathologic immune HIT was present, but this was undetectable by rapid standard immunoassay. Interestingly, the patient also had high IgE antibodies, and it is possible that HIT in this patient was either autoimmune or maybe unknown cross-reacting IgE-specific HIT antibody was present, which was undetectable by HIT-specific IgG ELISA. In autoimmune disorders, we have reported a rare case of IgA myeloma, with previously unknown pathological IgA-specific insulin antibody production, causing severe hypoglycemia. This IgA-insulin antibody-related hypoglycemia responded to anti-myeloma chemotherapy with the disappearance of IgA insulin antibodies [15] and no evidence of hypoglycemia. Likewise, it is postulated that high titer IgE- antibody $[16,17]$ may be cross-reacting with PF4 to cause HIT. It should be noted that eosinophilic myocarditis HIT patients did respond to Argatroban and high dose methylprednisone 
and Mycophenolate Mofetil (MMF) therapy for Eosinophilic myocarditis. It is suggested that eosinophilic myocarditis cases with thrombocytopenia and thrombosis should be monitored closely, and various PFA and other newly developed PF4 enhanced platelet activation assays, platelet factor 4-dependent P-selectin expression assay (PEA) and hybrid functional assays may diagnose HIT in these cases. In addition, future investigations into IgE antibodies may confirm or refute this hypothesis of cross-reacting PF4 binding IgE antibody-related HIT in Eosinophilic immunologic disorders.

A case with a past history of ITP in remission may suggest cross-activation of autoimmune HIT. Clinically, this causes therapeutic challenges. It is suggested to start non- heparin anticoagulants along with immunotherapy, to deal with this possible cross-over between ITP and HIT cases. There are case reports of COVID-19 complicated by immune thrombocytopenic purpura and internal jugular vein thrombosis [31] and also vaccine-related thrombotic thrombocytopenia syndrome $[9,18,32]$.

Our data indicate that mechanical heart valve and cardiac device patients have a relatively high incidence of HIT. This may suggest additional mechanical heart valve or cardiac device HIT trigger effect in post-cardiothoracic surgery patients. A similar mechanism is reported in patients with Arthroplasty in general and knee implants, in particular, showing activation of spontaneous, autoimmune HIT without heparin, LMWH, or fondaparinux [19-24].

Our findings of two cases of HIT in mechanical heart valves patients with a history of remitting and relapsing course on Argatroban is different from delayed-onset HIT $^{25}$ and persistent HIT [26]. This changing clinical course of relapsing HIT on Argatroban in mechanical heart valve may be resistant, refractory and autoimmune requiring different therapeutic options including IV Immunoglobulins, and longterm monitoring.

The diagnosis of HIT in cardiothoracic patients is challenging. HIT polyspecific PF-4 antibody IgGAM screening test remains non-specific. Routine usage of HIT IgG PF4 specific ELISA along with standard platelet functional assays SRA/HIPA as well as PF4- enhanced testing and hybrid PF4 [11-14] assays should be used to diagnose HIT in cardiothoracic patients. Our data show that standard iceberg model [11] of HIT diagnosis may not fit all these cardiothoracic patients. A modified diagnostic algorithm may be needed as illustrated in Figure 5A. Our results suggest that underlying cardiac immunologic disease, like sarcoid and eosinophilic myocarditis, has implications on laboratory diagnosis of HIT and its therapy $[27,28]$. Furthermore, HIT in cardiothoracic patients does not stop by stopping heparin. Pathologic HIT is continuing to evolve in cardiothoracic patients even after stopping Heparin, and on argatroban and the danger remains when it transforms from Heparin-related to spontaneous or immune or resistant HIT requiring a prompt change of therapy in deteriorating pathology. It was noted that patients with an underlying diagnosis of autoimmune disease, or with h/o treated ITP do not follow standard clinical course, and remitted ITP may have a cross-over relapse in autoimmune HIT with the negativity of HIT PF4-IgG antibody. All diagnostic approaches should be utilized to ascertain the diagnosis of autoimmune HIT in these cases [11-14]. This will guide in initiating appropriate and specific HIT therapy including Immunomodulatory drugs, IV immunoglobulins, and plasma exchange along with non-heparin agents $[27,28]$. Our findings could be criticized for lacking special PFA and evolving new PF4 enhanced assays in HIT IgG neg HIT and further advanced and specialized immunology tests in various remitting, relapsing, resistant and refractory cases and therefore, it may be speculative. However, this is a retrospective study and a possible confirmatory element in our findings is the chosen therapeutic option and appropriate clinical response of patients. It should be noted that heparin-induced thrombocytopenia is a rare disease, and the highest incidence is in cardiopulmonary bypass patients around $0.67 \%$. The risk with UFH is around 22 per 1000 and only 5 per 1000 with cases receiving LMWH. HIT with thrombosis (HITT) is significantly less, usually, 4 per 1000 with UFH and vs 17 per 1000 with LMWH [35]. Our case numbers may be low to draw a firm conclusion but our data does give an indication for the possible role of mechanical heart valves and cardiac devices in a high incidence of HIT in 16/80 cardiothoracic surgery patients from a single center. The HIT with thrombocytopenia and thrombosis is a rapidly evolving field [9,29,31,32], and more understanding of pathophysiology and use of new advanced diagnostic tests in new cases may provide further clarification.

A growing body of evidence suggests that not only heparin but also spontaneous autoimmune antibodies or unknown antibodies or orthopedic implant biomaterial or infections or vaccine can induce thrombocytopenia and thrombosis [9,19-24]. The role of mechanical heart valves and devices in triggering or aggravating or leading to spontaneous HIT syndrome requires further investigations. According to a 2009 published report from the United States, there were approximately 90,000 heart valves, half mechanical and half were bioprosthetic valves, replacements yearly [33]. Current online data research is showing over 182,000 heart valve implantations each year [34]. It could be argued that our findings in cardiothoracic patients related to HIT may be a caseload surgical practice effect and may be suggestive of selective surgery data bias. This is possible, but unlikely, given the multitude of cases in our cardiothoracic surgery, ICU and ECMO practice. It is suggested that the role of mechanical heart valves and cardiac devices in triggering or augmenting or progressing into spontaneous or remitting and relapsing autoimmune HIT is similar to the high incidence of HIT in patients with knee arthroplasty or heparin free HIT in knee implants surgery [19-24,29]. 


\section{Conclusion}

Our study indicates that 9/16 (> 50\%) HIT-positive patients had valve replacement or cardiac devices suggesting that like knee arthroplasty there is a high incidence of HIT in patients with mechanical heart valves and cardiac devices. A larger prospective study is needed to assess and understand HIT activation, progression, remitting, relapsing and refractory mechanisms and appropriate anticoagulation and immunotherapy options in cardiothoracic patients with immunological disorders, mechanical heart valves and mechanical cardiac devices.

\section{Acknowledgments}

Haematology BMS, N. Ahmed, S. Fox, F. Kamani, B. Mistry, S. Owen and others for Lab testing.

ITU/HDU colleagues: D. Hall, J. Mitchell, M. Scaramuzzi and all ITU and anesthesia team at Harefield Hospital for looking after and monitoring these complicated patients.

Cardiothoracic colleagues: T. Bahrami, M. Balakrishnan, O. Dar, F. de Robertis, F. Riesgo Gil and others for giving access to their patient's records.

Author's contribution: MHM contributed to patient care, analyzed laboratory and clinical data, and wrote the manuscript. KA and VJ did lab tests and collected lab data. EG, AH, PH contributed to ICU patient care, US and SKB contributed to surgical care. All authors contributed to manuscript reviewing.

Funding: NHS (National Health Service, England) UK.

Authors statement: The manuscript has been read and approved for submission by all authors.

Essentials: Non-immune causes of thrombocytopenia account for $80 \%$ of cases in the intensive care unit (ICU).

Pan-specific HIT antibody shows the positive predictive value of $35 \%$ with diagnostic uncertainty.

Remitting, relapsing and resistant HIT clinical course is observed in cardiothoracic cases.

A high incidence of HIT is observed in Mechanical heart valves surgery.

\section{References}

1. Greinacher A. Heparin-Induced Thrombocytopenia. N Engl J Med 2015; 373: 1883-1884.

PubMed: https://pubmed.ncbi.nlm.nih.gov/26535525/

2. Linkins LA, Dans AL, Moores LK, Bona R, Davidson BL, et al. Treatment and prevention of heparin-induced thrombocytopenia: Antithrombotic Therapy and Prevention of Thrombosis, 9th ed: American College of Chest Physicians Evidence-Based Clinical Practice Guidelines. Chest. 2012; 141: e495S-530S.

PubMed: https://pubmed.ncbi.nlm.nih.gov/22315270/
3. Cuker A, Gowthami MA, Chong BH, Cines DB, Greinacher A, et al. American Society of Hematology guidelines for management of venous thromboembolism: Heparin-induced thrombocytopenia. Blood Adv. 2018; 2: 3360-3392.

PubMed: https://pubmed.ncbi.nlm.nih.gov/30482768/

4. Warkentin TE, Greinacher A. Heparin-Induced Thrombocytopenia and Cardiac Surgery. Ann Thorac Surg. 2003; 76: 2121-2131. PubMed: https://pubmed.ncbi.nlm.nih.gov/14667668/

5. Everett BM, Yeh R, Foo SY, Criss D, Van Cott EM, et al. Prevalence of Heparin/Platelet Factor 4 Antibodies. Before and After Cardiac Surgery. Ann Thorac Surg. 2007; 83: 592-597. PubMed: https://pubmed.ncbi.nlm.nih.gov/17257993/

6. Squiccimarro E, Jiritano F, Serraino GF, ten Cate H, Paparella D, et al Quantitative and Qualitative Platelet Derangements in Cardiac Surgery and Extracorporeal Life Support. J Clin Med. 2021; 10: 615. PubMed: https://pubmed.ncbi.nlm.nih.gov/33561947/

7. Husseinzadeh HD, Gimotty PA, Pishko AM, Buckley M, Warkentin TE et al. Diagnostic accuracy of IgG-specific versus poly-specific enzymelinked immunoassays in heparin-induced thrombocytopenia: a systematic review and meta-analysis. J Thromb Haemost. 2017; 15: 1203-1212.

PubMed: https://pubmed.ncbi.nlm.nih.gov/28374939/

8. Sahu KK, Jindal V, Anderson J, Siddiqui AD, Jaiyesimi IA. Current Perspectives on Diagnostic Assays and Anti-PF4 Antibodies for the Diagnosis of Heparin-Induced Thrombocytopenia. J Blood Med. 2020; 11: 267-277.

PubMed: https://pubmed.ncbi.nlm.nih.gov/32884385/

9. Cines DB, Bussell JB. SARS-CoV-2 Vaccine-Induced Immune Thrombotic Thrombocytopenia. N Engl J Med. 2021; 384: 2254-2256. PubMed: https://pubmed.ncbi.nlm.nih.gov/33861524/

10. Pandya KA, Johnson EG, Davis GA, Padmanabhan A. Serotonin release assay (SRA) negative HIT: a newly recognized entity: implications for diagnosis and management. Thromb Res. 2018; 172: 169-171. PubMed: https://pubmed.ncbi.nlm.nih.gov/30419472/

11. Warkentin TE. Laboratory diagnosis of heparin-induced thrombocytopenia. Int J Lab Hematol. 2019; 41: 15-25. PubMed: https://pubmed.ncbi.nlm.nih.gov/31069988/

12. Padmanabhan A, Jones CG, Curtis BR, Bougie DW, Sullivan MJ, et al A novel PF4-dependent platelet activation assay identifies patients likely to have heparin-induced thrombocytopenia/ thrombosis. Chest. 2016; 150: 506-515.

PubMed: https://pubmed.ncbi.nlm.nih.gov/26905366/

13. Vayne C, Guery EA, Kizlik-Masson C, Rollin J, Bauters A, et al. Beneficial effect of exogenous platelet factor 4 for detecting pathogenic heparin-induced thrombocytopenia antibodies. $\mathrm{Br} \mathrm{J}$ Haematol. 2017; 179: 811-819.

PubMed: https://pubmed.ncbi.nlm.nih.gov/29048130/

14. Vayne C, Rollin J, Gruel Y, Pouplard C, Galinat H, et al. PF4 Immunoassays in Vaccine-Induced Thrombotic Thrombocytopenia. N Engl J Med. 385: 376-378.

PubMed: https://pubmed.ncbi.nlm.nih.gov/34010527/

15. Halsall DJ, Mangi, Soos M, Fahie-Wilson MN, Wark G, et al Hypoglycemia due to an Insulin Binding Antibody in a Patient with an IgA-Myeloma. J Clin Endocrinol Metab. 2007; 92: 2013-2016. PubMed: https://pubmed.ncbi.nlm.nih.gov/17405845/

16. Poulsen LK, Jensen BM, Esteban V, Garvey LH. Beyond IgE-When Do IgE-Crosslinking and Effector Cell Activation Lead to Clinical Anaphylaxis? Front Immunol. 2017; 8: 871.

PubMed: https://pubmed.ncbi.nlm.nih.gov/28848540/

17. Thi-Huong, Nguyen TH. Not Only Heparin but Also Antibody Induces thrombocytopenia. Book Chapter 3. 2018.

18. Ahmed M, Habis S, Samotwoka M, Mahmoud A, Chin M. Mesenteric Ischemia Caused by Heparin-induced Thrombocytopenia: A Case 
Report. Cureus. 2019; 11: e3900.

PubMed: https://pubmed.ncbi.nlm.nih.gov/30911456/

19. Greinacher A. Me or not me? The danger of spontaneity. Blood. 2014 123: 3536-3538.

20. Warkentin TE. Knee replacement and HIT without heparin: Blood. 2016; 127: 961-962.

21. Bito S, Miyata S, Migita K, Nakamura M, Shinohara K, et al. Mechanical prophylaxis is a heparin-independent risk for antiplatelet factor 4/ heparin antibody formation after orthopedic surgery. Blood. 2016; 127: 1036-1043.

PubMed: https://pubmed.ncbi.nlm.nih.gov/26659923/

22. Burch M, Cooper B. Fondaparinux-associated heparin-induced thrombocytopenia. Proc (Bayl Univ Med Cent). 2012; 25: 13-15. PubMed: https://pubmed.ncbi.nlm.nih.gov/22275775/

23. Olevsky O, Rosove MH. Spontaneous Heparin-Induced Thrombocytopenia and Thrombosis After Total Knee Arthroplasty: A Report of 2 Cases. Arthroplasty Today. 2021; 9: 50-52.

PubMed: https://pubmed.ncbi.nlm.nih.gov/34026990/

24. Swarup S, Kopel J, Yendala R, Thirumala S, Quick DP. Spontaneous Heparin-Induced Thrombocytopenia HIT following curettage and bone graft of femur in a patient with monostatic fibrous dysplasia. J Thrombosis Res. 2020; 196: 75-77.

PubMed: https://pubmed.ncbi.nlm.nih.gov/32853979/

25. Warkentin TE, Kelton JG. Delayed-onset heparin-induced thrombocytopenia and thrombosis. Ann Intern Med. 2001; 135: 502-506. PubMed: https://pubmed.ncbi.nlm.nih.gov/11578153/

26. Kopolovic I, Warkentin TE. Progressive thrombocytopenia after cardiac surgery in a 67-year-old man. CMAJ. 2014; 186: 929-933.

PubMed: https://pubmed.ncbi.nlm.nih.gov/24756626/

27. Padmanabhan A, Jones CG, Pechauer SM, Curtis BR, Bougie DW, et al. IVIg for treatment of severe refractory heparin-induced thrombocytopenia. Chest. 2017; 152: 478-485.

PubMed: https://pubmed.ncbi.nlm.nih.gov/28427966/

28. Warkentin TE, Anderson JAM. How I treat patients with a history of heparin-induced thrombocytopenia. Blood. 2016; 128: 348-359. PubMed: https://pubmed.ncbi.nlm.nih.gov/27114458/

29. Warkentin TE, Greinacher A. Spontaneous HIT syndrome: Knee replacement, infection, and parallels with vaccine-induced immune thrombotic thrombocytopenia. Thromb Res. 2021; 204: 40-51. PubMed: https://pubmed.ncbi.nlm.nih.gov/34144250/

30. Ali K, PalomoLopez N, Jeyakumar V, Hurtado Doce A, Mangi M. Unusual laboratory features and clinical course of Heparin induced thrombocytopenia in cardiothoracic patients. EHA 2021 virtual congress 9-17 June 2021, abstract presentation. Hemasphere. 2021; S52: 543-a.

31. Bucke D, Alizadeh K, Hallam S. COVID-19 complicated by immune thrombocytopaenic purpura and internal jugular vein thrombosis. BMJ Case Rep. 2021; 14: e243680.

PubMed: https://pubmed.ncbi.nlm.nih.gov/34285032/

32. Thrombosis with Thrombocytopenia Syndrome (also termed Vaccineinduced Thrombotic Thrombocytopenia). 2021.

https://www.hematology.org/covid-19/vaccine-induced-immunethrombotic-thrombocytopenia

33. Pibarot P, Dumesnil JG. Prosthetic Heart Valves Selection of the Optimal Prosthesis and Long-Term Management. Circulation 2009; 119: 1034-1048.

PubMed: https://pubmed.ncbi.nlm.nih.gov/19237674/

34. https://idataresearch.com/over-182000-heart-valve-replacements-peryear-in-the-united-states/

35. Hogan M, Berger SJ. Heparin-induced thrombocytopenia (HIT): Review of incidence, diagnosis, and management. Vascu Med. 2020; 25: $160-173$.

PubMed: https://pubmed.ncbi.nIm.nih.gov/32195628/ 